\title{
Shock layer instability near the Newtonian limit of hypervelocity flows
}

\author{
H. G. Hornung ${ }^{\text {a) }}$ \\ Graduate Aeronautical Laboratories, California Institute of Technology, MS 105-50, Pasadena, \\ California 91125 \\ P. Lemieux \\ Exponent, Inc., 5401 McConnell Avenue, Los Angeles, California 90066
}

(Received 13 February 2001; accepted 15 May 2001)

\begin{abstract}
The curved bow shock in hypersonic flow over a blunt body generates a shear layer with smoothly distributed vorticity. The vorticity magnitude is approximately proportional to the density ratio across the shock, which may be very large in hypervelocity flow, making the shear layer unstable. A computational study of the instability reveals that two distinct nonlinear growth mechanisms occur in such flows: First, the vortical structures formed in the layer move supersonically with respect to the flow beneath them and form shock waves that reflect from the body and reinforce the structures. Second, the structures deform the bow shock, forming triple points from which shear layers issue that feed the main shear layer. Significant differences exist between plane and axisymmetric flow. Particularly rapid growth is observed for free-stream disturbances with the wavelength approximately equal to the nose radius. The computational study indicates that the critical normal shock density ratio for which disturbances grow to large amplitudes within a few nose radii is approximately 14 . This served as a guide to the design of a physical experiment in which a spherical projectile moves at high speed through propane or carbon dioxide gas. The experiment confirms the approximate value of the critical density ratio, as well as the features of the computed flows. Comparisons of calculations of perfect gas flows over a sphere with shadowgraphs of the projectile show very good agreement. The Newtonian theory of hypersonic flow, which applies at high density ratio, makes the assumption that the flow remains smooth. The results show that high density ratio also causes this assumption to fail. (C) 2001 American Institute of Physics.

[DOI: $10.1063 / 1.1383591]$
\end{abstract}

\section{INTRODUCTION}

A curved shock wave in a steady, uniform free stream generates vorticity

$$
\omega=U_{\infty} \kappa \cos \beta\left(1-\rho_{\infty} / \rho\right)^{2} \rho / \rho_{\infty}
$$

by the baroclinic mechanism within the shock wave (see Lighthill, ${ }^{1}$ and Hayes and Probstein ${ }^{2}$ ). Here, $U_{\infty}, \rho_{\infty}$ are free-stream speed and density, and $\beta, \kappa$ are the shock angle and curvature at the point of interest. In hypervelocity flow of a molecular gas over a blunt body the post-shock temperature is so high that substantial dissociation occurs. One consequence is that the endothermic dissociation process absorbs a large fraction of the thermal energy of the gas, so that the temperature falls dramatically and the density rises correspondingly. Thus the density ratio, $\rho / \rho_{\infty}$, across the shock and (thin) reaction zone is very large. For example, in air flows at $7 \mathrm{~km} / \mathrm{s}$, a density ratio of 12 is typical, while in carbon dioxide flows, such as occur, e.g., in traversing the atmosphere of Mars, the density ratio may be as high as 28 . This is the kind of flow of interest here. With such high density ratio, the inverse of the ratio may be neglected compared with 1 in Eq. (1).

a) Telephone: 626-395-4551; fax: 626-449-2677; electronic mail: hans@galcit.caltech.edu
Consider Eq. (1) in connection with flow over a blunt body. At the normal shock point, $\omega$ is zero, because the shock angle is $90^{\circ}$. Far downstream, where (on an asymptotically slender body) the shock approaches a straight Mach wave, the density ratio becomes one and the curvature goes to zero, so $\omega$ again vanishes. Somewhere between these limits a maximum value of $\omega$ must therefore occur. With typical shock shapes this maximum lies at approximately $\beta=60^{\circ}$. Since the shock angle and curvature vary smoothly with distance along the shock, a smooth distribution of vorticity results. The shock generates a shear layer with a smooth vorticity maximum. Since the flow is compressible, the vorticity does not remain constant along streamlines even in the absence of diffusion, but is influenced by the density and pressure fields.

The stability of a shear layer with distributed vorticity has been studied extensively (see, e.g., Drazin and Reid, ${ }^{3}$ or, more relevant to the present investigation, Zhuang et $\mathrm{al}^{4}{ }^{4}$ ). A qualitative feature is that, other things being equal, layers with higher maximum vorticity and smaller thickness are more unstable (a result that follows already from scaling arguments). Not only does the high density ratio of hypervelocity flows cause proportionately higher maximum vorticity in the layer, but it also causes the shock layer, and therefore the shear layer, to be proportionately thinner. It may therefore be expected that the shear layer that results from shock 
curvature in hypervelocity flows becomes unstable at sufficiently high density ratio.

The instability of the layer has been considered by aerodynamicists for some time as a cause of boundary layer transition. This is relevant, because, far downstream the shear layer will eventually be ingested by the growing boundary layer. Since the shear layer represents the edge of a highentropy layer close to the body, this is also known as entropy-layer swallowing (see, e.g., Stetson et al. ${ }^{5}$ ). There is a significant difference between inviscid flow in axial and plane symmetry in this phenomenon. The reason is that, with axial symmetry, the stretching term in the vorticity equation causes the vorticity on the body surface to be finite, while it is zero in plane symmetry; see Hayes and Probstein. ${ }^{2}$ The result is that the shear layer is further from the body in plane flow so that, as regards entropy-layer swallowing, plane and axisymmetric flows behave very differently.

At a curved shock, vorticity is imparted to a material element. Even in plane flow, the vorticity of the material element is subsequently changed by the fact that the density and pressure of the material element change. In the particular case of homenthalpic, plane, steady flow of a perfect gas, the ratio of vorticity to pressure is conserved along a streamline (see, e.g., Vaszonyi ${ }^{6}$ ). Thus if the pressure is constant along a streamline, then so also is the vorticity. A relatively simple configuration for an exploratory study of the instability of a shear layer in the shock layer of a blunt body in hypersonic flow is therefore a hemicylindrically blunted wedge. This has the necessary curved bow shock followed by a constantpressure region downstream. To illustrate the behavior of the shear layer generated in such a flow, Fig. 1 shows an example of an Euler computation of flow over a cylindrically blunted $30^{\circ}$ wedge. Note the location of the region of maximum shear relative to the body. In plane flow, the shear layer remains at a constant distance from the wedge surface, and the growth of the shock layer outside of the shear layer leaves the shear layer virtually unchanged.

In the following, we therefore start with a computational study of the stability of the shear layer in such a flow. A disturbance is introduced to the flow by imposing an unsteady boundary condition at the inflow boundary. This study is then followed by a similar one on axisymmetric flow, to examine the difference in behavior discussed earlier. Finally, the experience gained from the computational studies is used to design a physical experiment. It turns out that in the latter it is necessary to choose a differently shaped model. Computations of this new configuration are therefore added to compare with the physical flow.

\section{COMPUTATIONAL EXPERIMENT}

\section{A. The computational setup}

For the computations, the software system Amrita, constructed by James Quirk (see Quirk ${ }^{7}$ ) was used. Amrita is a system that automates and packages computational tasks in such a way that the packages can be combined (dynamically linked) according to instructions written in a high-level scripting language. The present application uses features of Amrita that include the automatic construction of different
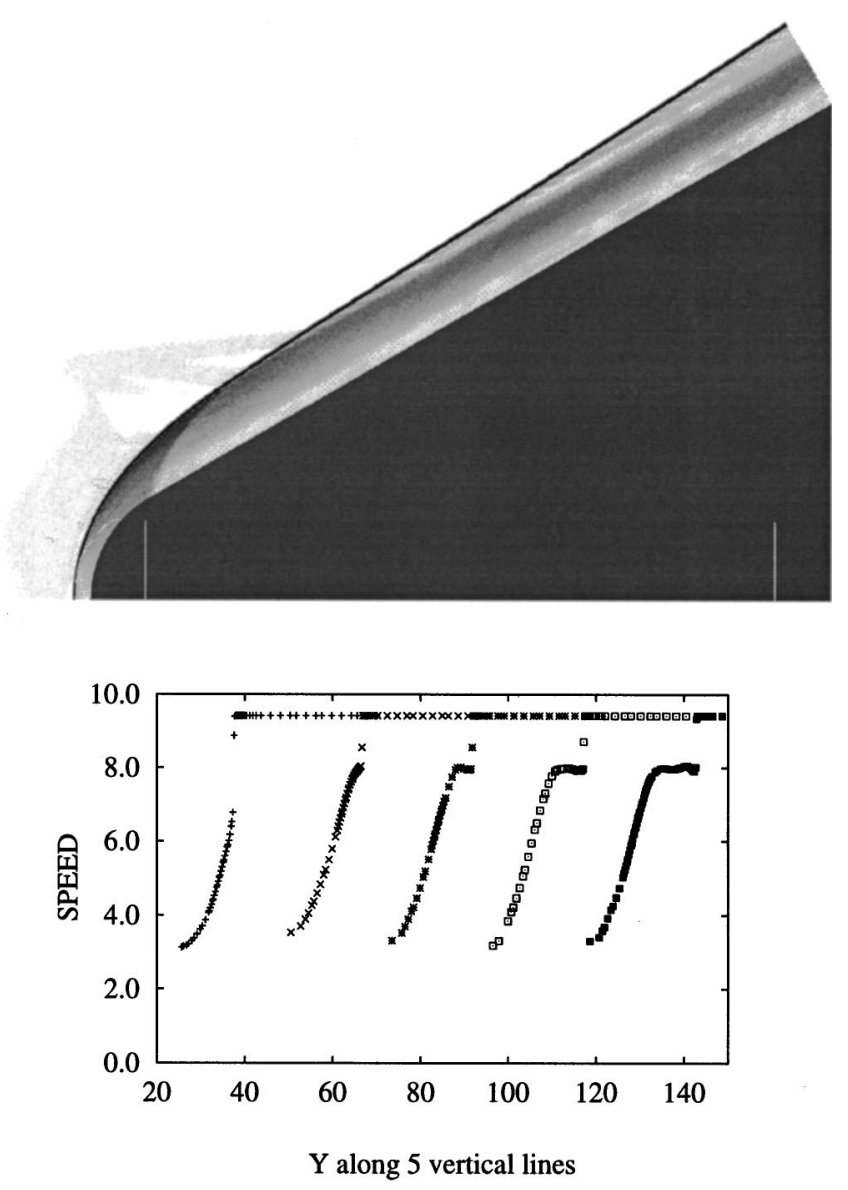

FIG. 1. Top: Pseudo-schlieren image of computed flow over cylinderwedge, $M_{\infty}=9, \gamma=1.095$, making the normal shock density ratio 17.5. Grayshading is a monotonic function of the magnitude of the fractional density gradient. The dark region corresponds approximately to the shear layer. The white line in the flow field is the sonic line. Bottom: Five profiles of speed across the layer uniformly spaced between the two white lines show that the shear layer does not change significantly with downstream distance.

Euler solvers, automatic documentation of the codes, automatic adaptive mesh refinement according to simply chosen criteria, and scripting-language-driven computation, archiving, and post-processing of the results. The automation of the assembly and sequencing of the tasks makes for drastically reduced possibility of hidden errors. More importantly, it makes computational investigations transparent and testable by others. The ability to change one package at a time, without changing the rest of the scheme, permits easy detection of sources of error. The scope of the software system far exceeds its use here.

Adaptive mesh refinement was applied in two stages of a factor of 3 each, on a coarse grid of $40 \times 240$ cells, so that the effective grid was $360 \times 2160$. The grid refinement criterion was a threshold value of the magnitude of the density gradient. Extensive tests were performed on different criteria and degrees of grid refinement in order to ensure that the phenomena observed were not grid-resolution related features.

In most of the following, a flux-limited, operator-split solver is used, with a body-fitted grid. With such a scheme, nonuniformity of the grid introduces numerical noise in the flow. The maximum magnitude of this noise in density is 


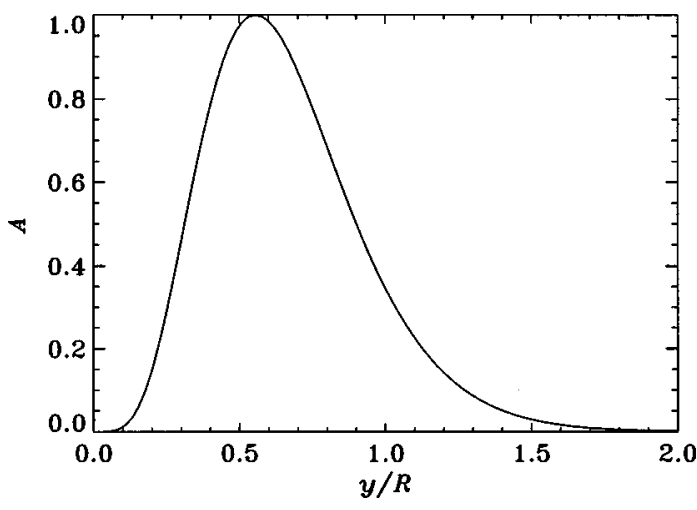

FIG. 2. Form of the amplitude function of the perturbation.

$0.3 \%$ of the free-stream density in our case. Provided that the normal shock density ratio is small enough, this noise is insufficient to make the flow unstable, as in the example shown in Fig. 1. Such stable flows can then be excited with deliberately introduced disturbances, whose growth can be studied to determine the instability conditions.

\section{B. Choice of disturbance}

A suitable disturbance for excitation of instability in a shear layer is a periodic variation of the velocity component normal to the shear layer: If such a disturbance is introduced all along the inflow boundary of the computational domain, it causes trouble at the plane or axis of symmetry. We therefore choose an amplitude function, $A$, for the disturbance, which is of the form

$$
V / U_{\infty}=A(y / R) \sin \left(2 \pi U_{\infty} t / \lambda\right),
$$

where $V$ is the $y$-component of velocity, $R$ is the nose radius, $t$ is time, and $\lambda$ is the wavelength of the disturbance. The amplitude function is

$$
A(y / R)=C_{1}(y / R)^{5} \exp \left(-C_{2} y / R\right) .
$$

The numerical constants $C_{1}$ and $C_{2}$ can then be chosen to adjust the magnitude of the disturbance and the $y$-location of its maximum. A plot of $A$ is presented in Fig. 2.

This is not the only type of disturbance that was applied to the problem. Others included unsteady density and pressure conditions at the inflow boundary. The behavior of each disturbance type was tested by applying it to an otherwise uniform flow. As expected, the disturbances steepen into weak shock waves on their forward side, but with the small amplitudes introduced here, visible steepening only occurred after large distances, and, for sufficiently small disturbances, effects of numerical dissipation prevent the steepening. In all the cases studied here, the disturbances decay slightly over the small distance between the inflow boundary and the bow shock.

As an example of the effect of a disturbance on the flow shown in Fig. 1, a disturbance with maximum amplitude $a$ $=V_{\max } / U_{\infty}=1.0 \%$ and with maximum located at $y / R$ $=0.556$ was switched on at the left boundary after the flow had reached steady state. This was then repeated with differ-

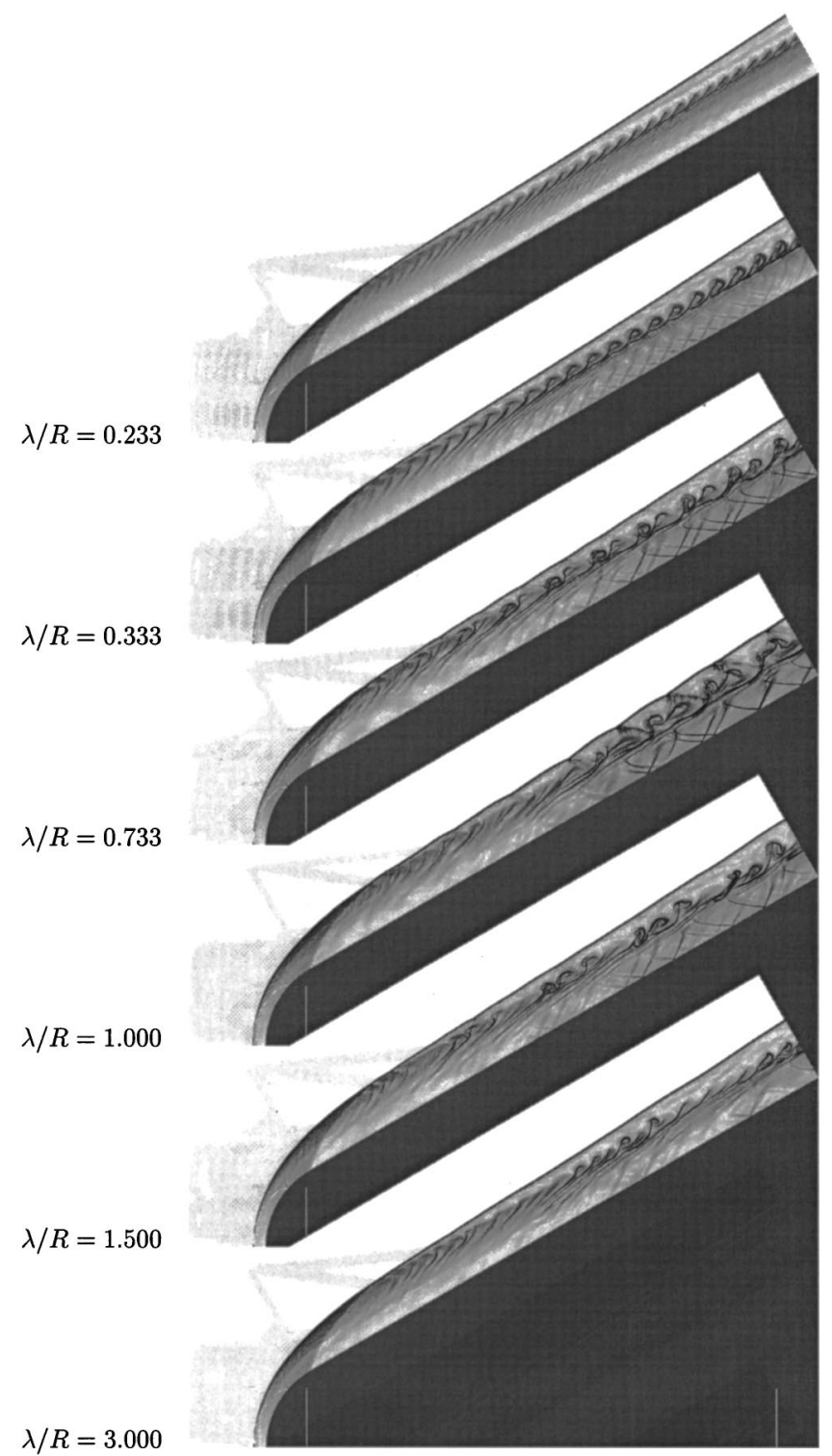

FIG. 3. Pseudo-schlieren images of the flow of Fig. 1, after a disturbance of maximum amplitude $1.0 \%$ is imposed at the left boundary. Note that the structures in the shear layer move supersonically and push shock waves that reflect from the wall. At large wavelengths the structures do not become larger, but form in packages spaced at the imposed wavelength.

ent disturbance wavelengths. A selection of the resulting pseudo-schlieren images is presented in Fig. 3.

A number of features of the growth of the disturbance emerge. At small wavelength $(\lambda / R=0.233)$ the disturbance is only slightly amplified. However, as the wavelength is increased, the growth is so strong that the structures, which move at supersonic speed relative to the flow between the shear layer and the body, push oblique shock waves into it, which are reflected from the wall and reimpinge on the shear layer.

Another feature is that the preferred wavelength that grows is quite small. In unconfined shear layers this preferred wavelength amounts to several shear-layer thicknesses, while it is of the order of the shear-layer thickness here. Also, the structures become so strong at $\lambda / R=1.0$ that they perturb the shock wave significantly. For wavelengths 


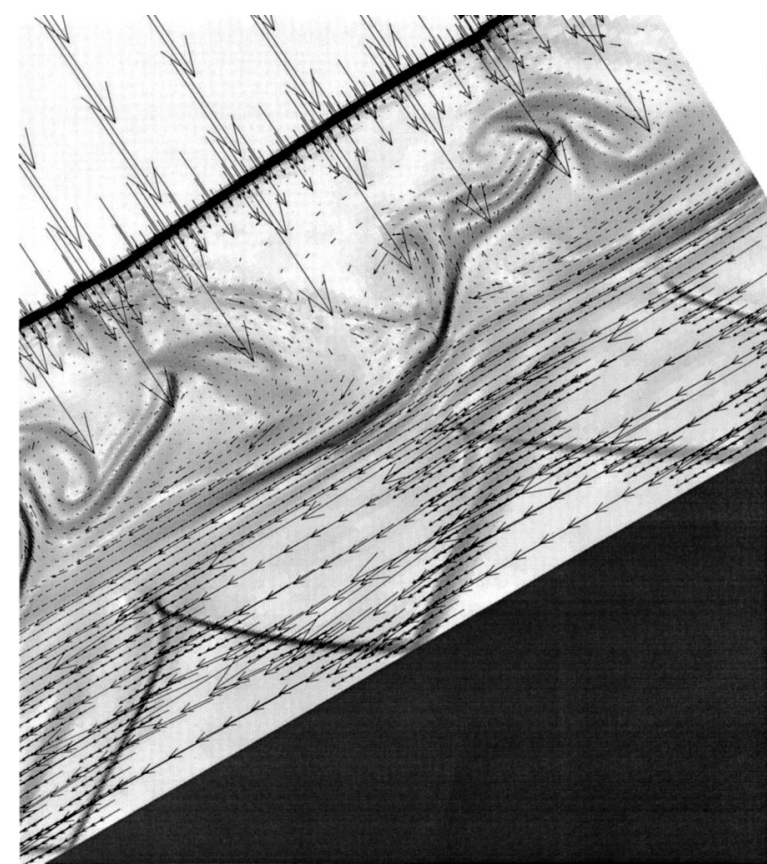

FIG. 4. Enlargement of portion of the flow in Fig. 3, case $\lambda / R=1.0$, showing vectors of velocity relative to a point approximately in one of the shearlayer structures. The length of the velocity vectors is proportional to the velocity, but the refined-grid vectors are plotted to a smaller scale. Note that the vectors show the vortical nature of the structures and identify the dark oblique lines underneath them as shock waves. Note also that the structures move approximately at the shock-parallel component of the free-stream speed.

that are larger than the preferred wavelength, the imposed wavelength appears in the form of packets of the small wavelength spaced at the imposed wavelength. At larger wavelengths the growth rate is strongly reduced again. A quantitative analysis shows that, in the flows presented in Fig. 3, the density fluctuations that result in the shear layer reach values up to $20 \%$ after a distance of a few nose radii.

Figure 4 shows an enlarged view of portion of Fig. 3, case $\lambda / R=1.0$, with vectors of velocity relative to the velocity of a point moving with one of the structures. The size of the vectors is proportional to this relative velocity (but refined-grid arrows are correspondingly smaller). Three features are brought out by this. First, the shear layer structures move with a speed close to the post-shock speed and they are vortical in nature. Second, the flow below the shear layer is very fast in this reference frame, explaining the occurrence of the oblique shocks that reflect from the wall. Third, when the disturbance has grown to such a degree that the shock becomes perturbed, weak triple points form on the shock (top left of Fig. 4), from which thin shear layers emerge that feed the main shear layer with even more vorticity of the same sign. Once this mechanism sets in, as computations at higher density ratios show, the growth of the disturbance enters a catastrophic phase that eventually produces a turbulent shock layer bounded toward the free stream by an irregular unsteady shock.

\section{Flow over a sphere-cone}

In axisymmetric flow, the vorticity of a material element at constant pressure does not remain constant, because the
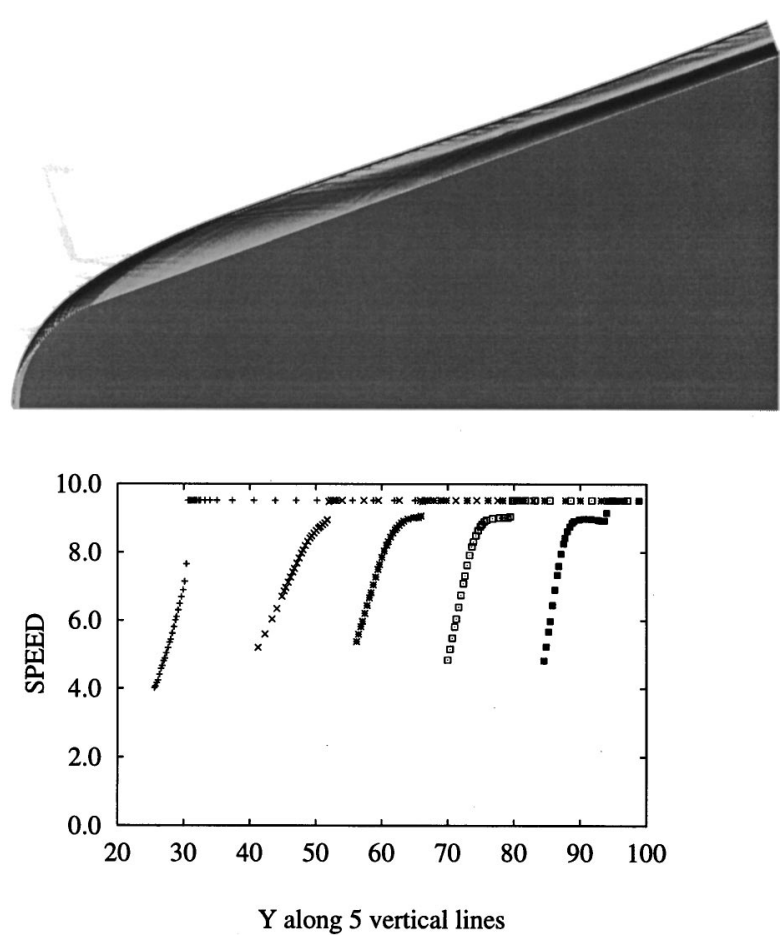

FIG. 5. Top: Pseudo-schlieren image of computed flow over a $20^{\circ}$ spherecone, $M_{\infty}=9, \gamma=1.12$, corresponding to a normal shock density ratio of 14.7. The body-fitted grid represents curved surfaces as polygons. On the spherical nose this causes waves to be emitted from the surface. These are significantly stronger in this axisymmetric case, because the polygons lie in a higher-Mach-number part of the flow. The waves are reflected from the shock and again from the body a couple of times. Although they introduce a significant disturbance they are not strong enough to cause the shear layer to become unstable at this density ratio. Bottom: Five uniformly spaced profiles of speed across the layer. Note how the vorticity of the shear layer increases with downstream distance, as a consequence of the vortex lines being stretched.

vortex lines may be stretched. Thus in the analogous model to that of the cylinder-wedge of plane flow, namely a spherecone, the vorticity of a material element will increase with time. This may be seen in the no-disturbance computation presented in Fig. 5. Observe the profiles of speed across the shock layer. From the second profile onward, the vorticity (the slope of the velocity profile) increases with downstream distance and the region of maximum vorticity is at or very near the body. Thus the shear layer and any structures that might develop in it will not move at a supersonic speed relative to the region between the shear layer and the body, and we may expect this region to be free of shock waves.

In this flow field a feature of blunt cone and blunt wedge flows may be observed: The shock shape near the nose is like that of a slender blunt body, i.e., like the paraboloid (in axisymmetric flow) of the blast wave analogy, which would intersect the cone far downstream. However, far downstream, where the flow becomes asymptotically like that over a sharp cone, the shock shape has to become conical, so that the shock exhibits a point of inflection.

Computations with deliberately introduced disturbances were also made in the axisymmetric case for density ratios of 14.7 and 17.5. These are presented in Figs. 6 and 7. 


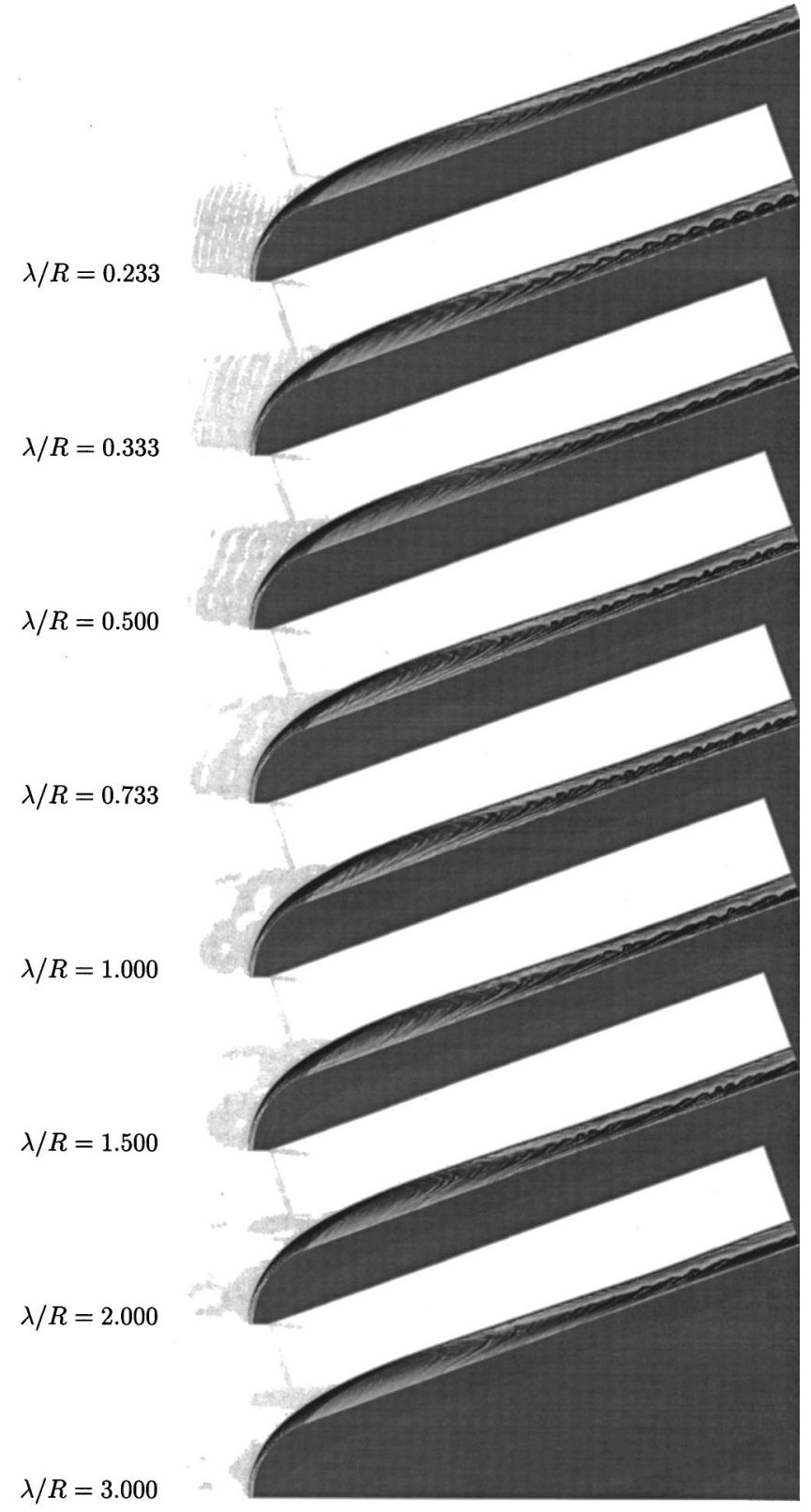

FIG. 6. Pseudo-schlieren images of flow over a sphere-cone at $M_{\infty}=9, \gamma$ $=1.12$, making the normal shock density ratio 14.7 , with the same freestream disturbance as in the case of the cylinder-wedge.

\section{The critical density ratio}

As was pointed out earlier, the shear layer thickness decreases and its intensity increases in approximate proportion to the normal shock density ratio. Both of these increase the instability of the shear layer. In an inviscid flow the shear layer will eventually exhibit instability far downstream, of course, but a certain critical density ratio is likely to exist, below which no detectable evidence of instability exists within the computational domain, and above which it does. A large number of computations were made in both the plane and the axisymmetric flows with different disturbance amplitudes and different values of Mach number and specific heat ratio, in order to examine the influence of the normal shock density ratio on the stability.

When the normal shock density ratio was smaller than

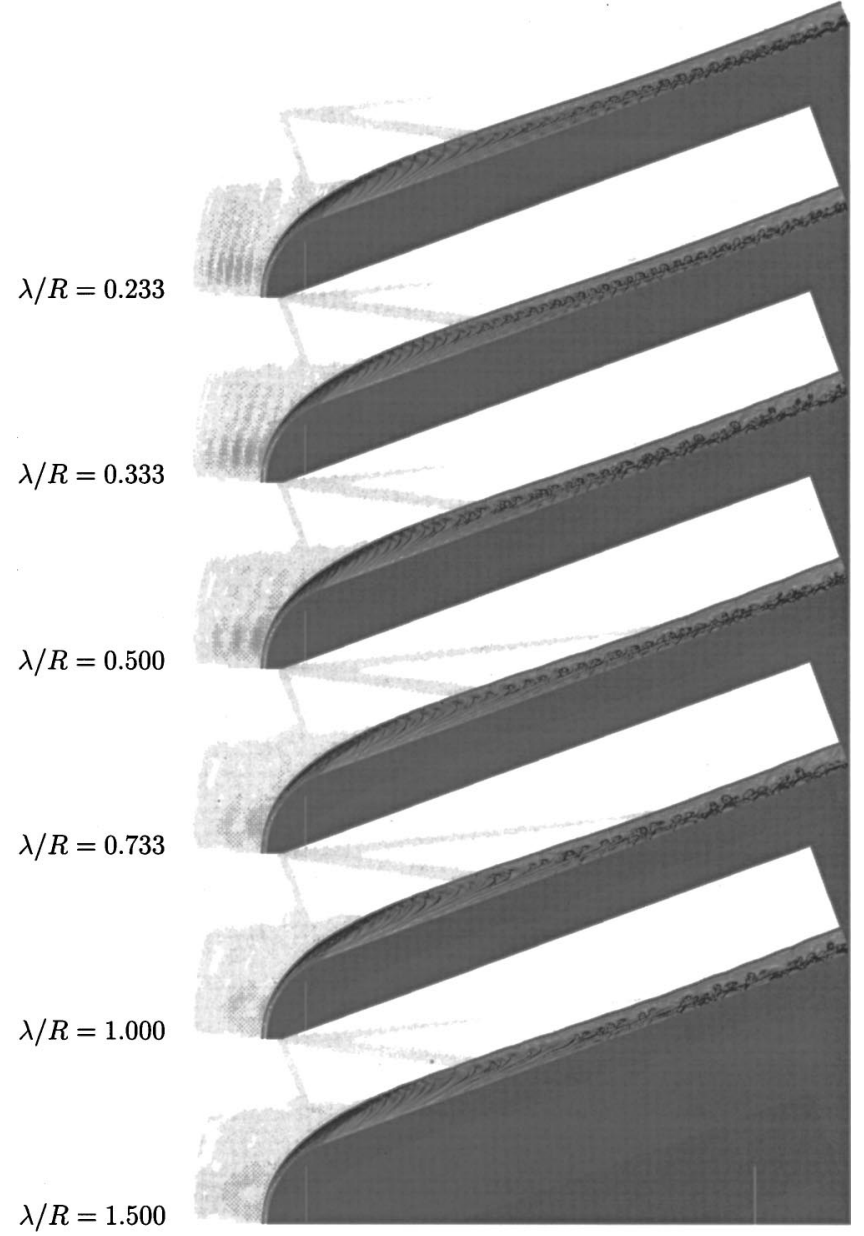

FIG. 7. Pseudo-schlieren images of flow over a sphere-cone at $M_{\infty}=9, \gamma$ $=1.095$, (normal shock density ratio 17.5) with the same free-stream disturbance.

11 , disturbance amplitudes as large as $2 \%$ did not exhibit any growth of structures in the shear layer. On the other hand, when the normal shock density ratio was 18 or greater, it was not necessary to introduce a disturbance at all in order to cause the shear layer to become unstable within the computational domain. This indicates that the small nonuniformities in the free stream that result from the nonuniformity of the body-fitted grid (density perturbations of up to $0.3 \%$ of the free-stream density) suffice to trigger the instability of the shear layer within the computational domain. This therefore suggests that, in an experiment to test the results of the exploratory computational study, normal shock density ratios of at least 14 , and preferably more should be achievable.

\section{THE PHYSICAL EXPERIMENT}

An example of a situation in which high normal shock density ratios occur is hypervelocity flight in a carbondioxide atmosphere. One might expect that such flows could be achieved in a hypervelocity test facility such as a freepiston shock tunnel, where flow speeds are achievable that produce very significant dissociation (and therefore density 


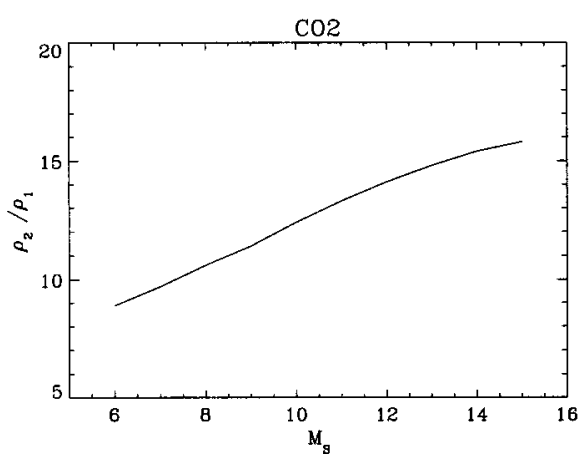

increase) downstream of the bow shock wave of a blunt body. Unfortunately, this is not possible in a reflected shock tunnel, for the following reasons:

In a reflected shock tunnel, the high-speed flow is generated by converting the thermal energy of a stationary reservoir gas into ordered kinetic energy of flow by means of a nozzle expansion. The thermal energy of the stationary gas necessarily has to be comparable to the dissociation energy of the gas if significant dissociation is to occur after the bow shock. This means that the reservoir gas is partially dissociated. During the passage of the gas through the nozzle, some recombination does occur, but as the density decreases, there comes a point when the composition freezes. Thus the flow that arrives at the bow shock is already partially dissociated, so that the amount of dissociation that occurs after the bow shock (and therefore the amount of density increase) is smaller than would be observed in flow with an undissociated free stream. It turns out that the highest normal shock density ratio achievable in the T5 free-piston shock tunnel at Caltech is approximately 12. Experiments involving flow over a cylinder-wedge such as those studied in the exploratory computational experiment yielded only very scant evidence of instability in the shear layer; see Lemieux. ${ }^{8}$

An alternative to generating flow over a stationary model is to shoot a projectile into low-temperature stationary gas. A modification of T5, designed and built by Bélanger and Kaneshige, ${ }^{9}$ converts the facility into a light gas gun with which a $25 \mathrm{~mm}$ diameter projectile can be accelerated to 3 $\mathrm{km} / \mathrm{s}$ and shot into a test section containing any gas between two thin mylar diaphragms. If a density ratio of 16 is to be achieved by shooting a projectile into carbon dioxide, the velocity of the projectile has to be of the order of $4 \mathrm{~km} / \mathrm{s}$. However, high density ratios can be achieved more easily by using a gas with a very small ratio of specific heats. For example, with propane $\left(\mathrm{C}_{3} \mathrm{H}_{8}\right)$, a normal shock density ratio of 17 can be achieved at $2.8 \mathrm{~km} / \mathrm{s}$; see Fig. $8 .{ }^{10}$

\section{A. Experimental setup}

In the light gas gun modification of T5, a gun barrel is attached to the end of the shock tube, which reaches approximately to the middle of the dump tank. A test section attached to the far end of the dump tank contains the test gas between thin mylar diaphragms. The shock-heated and compressed helium in the shock tube serves as the driver that propels the projectile along the gun barrel, through the dump tank, and into the test section. As the projectile travels through the test section, shadowgraph records of the flow may be taken through a window. A drawing of the test section is shown in Fig. 9.

To measure the projectile speed, three photodetectors and three pressure transducers are installed in the test section, as shown in Fig. 9. The projectile breaks a wire, providing the first timing signal for the speed determination. The photodetector signals are used together with this to calculate the projectile speed. The projectile speed can be varied from 1 to $3 \mathrm{~km} / \mathrm{s}$ by changing the gas gun driver gas and conditions. At the higher speeds, it was found that nylon did not have sufficient impact strength to survive, and it was necessary to switch to the much tougher material Lexan.

\section{B. Experimental results}

At conditions where the normal shock density ratio is less than 14, the flow over the projectile is characterized by a smooth shock wave. Two examples are shown in the shadowgraphs of Figs. 10 and 11 for propane and carbon dioxide flows, respectively. In contrast, when the conditions are such that the density ratio is larger than 15 (see Figs. 12 and 13), the shock wave is significantly perturbed, so much so that the features of the flow in the central wake are completely obscured by the refraction of the light in the outer part of the axisymmetric flow in the (line-of-sight integrating) shadow-

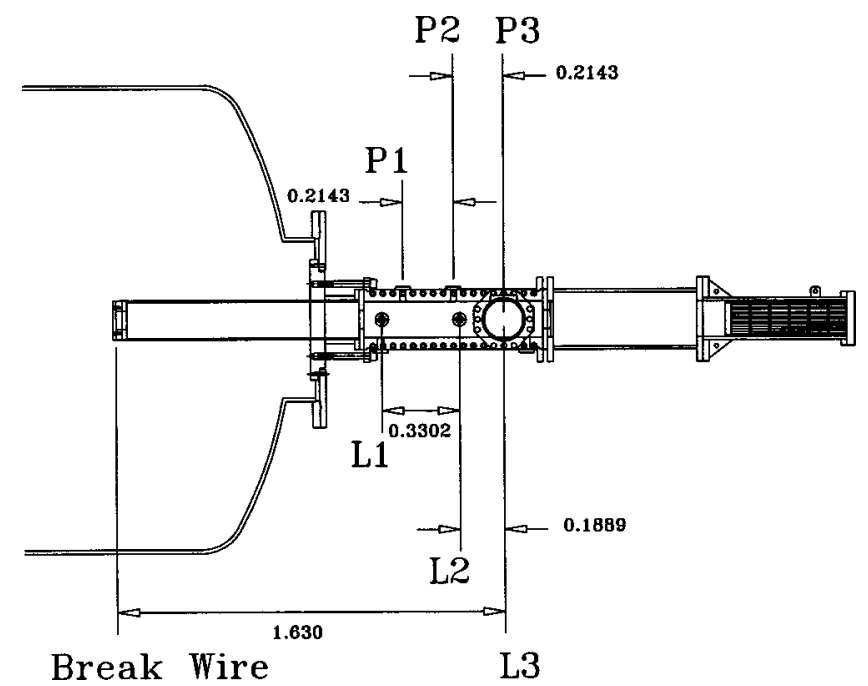

FIG. 9. Light gas gun test section. Photodetector and pressure transducer stations are labeled L and P, respectively. Dimensions shown are in meters. The stations P3 and L3 are in the middle of the window. 


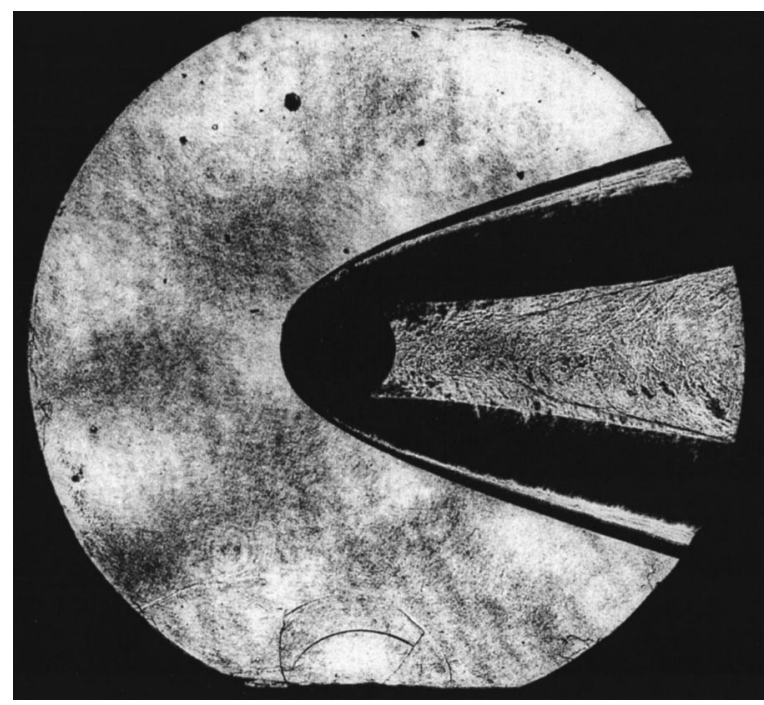

FIG. 10. Shadowgraph of projectile moving at $2.26 \mathrm{~km} / \mathrm{s}$ in propane, giving a normal shock density ratio of 13 . The black regions just inboard from the shock result from the very large separation of the elements of the shadowgraph system. This causes regions of large refractive index gradient to refract the beam so much that it misses one of the mirrors completely, causing that region to be black. The shock front is quite smooth, and turbulence is only evident in the central wake. The wake shocks are faintly visible.

graph. This shock wave perturbation looks similar to that of the second nonlinear amplification mechanism discussed in connection with the computations.

It is clear from these results that a dramatic change occurs in these flows in the range of normal shock density ratio around 14. In order to relate this to the phenomena that occur in the exploratory computational experiment, it is necessary to compute flows that are more nearly like the ones in the experiment.

It should be pointed out that a similar experimental observation of instability of high Mach number flow over a sphere has been made by Tumakaev, ${ }^{11}$ who attributes the

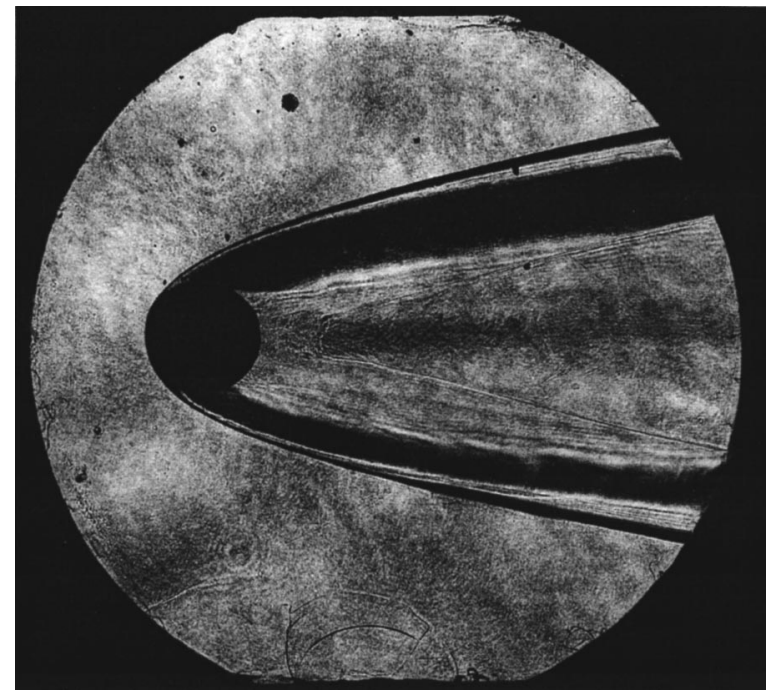

FIG. 11. Shadowgraph of projectile moving at $2.7 \mathrm{~km} / \mathrm{s}$ in carbon dioxide, giving a normal shock density ratio of 12 . Again the shock wave is smooth, and the features are much like those of Fig. 10.

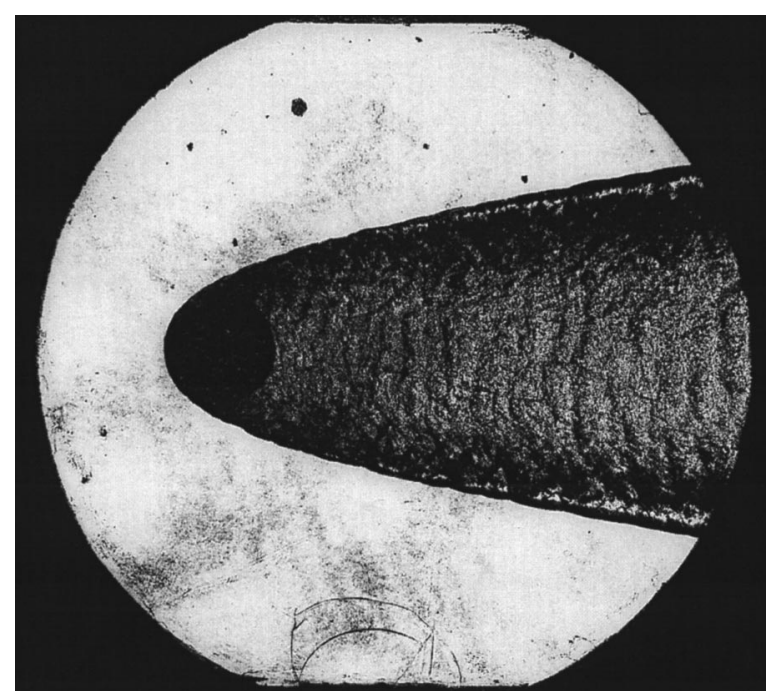

FIG. 12. Shadowgraph of projectile moving at $2.7 \mathrm{~km} / \mathrm{s}$ in propane, giving a normal shock density ratio of 20 . Note the dramatic perturbation of the shock wave, which manifests itself in features that stretch across the flow, obscuring the features of the central wake.

phenomenon to phase transitions. In our experiments no phase transitions occur, and we suspect that the phenomenon he observed also arises because of the shear layer instability that attends the high normal shock density ratio in his freon flows.

\section{COMPARISON WITH COMPUTATION OF FLOW OVER A SPHERE}

In the experiment, the gases exhibit high-temperature real-gas effects, in particular, vibrational excitation. We simulate the flow over a sphere at conditions that are as nearly the same as those in the experiment, but with a perfect gas, by making the density ratios of the computations the same as those in the experiment. Since the density ratio de-

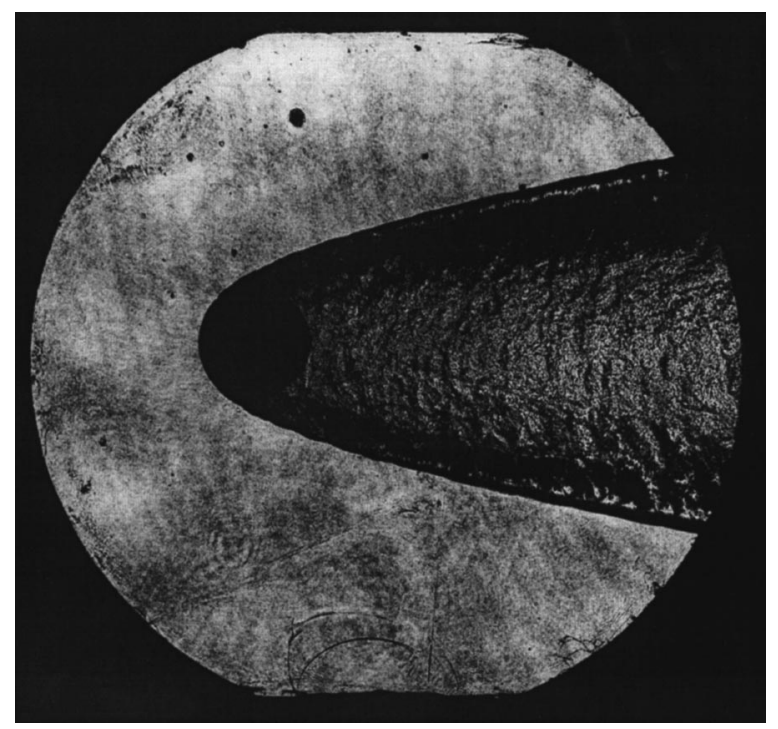

FIG. 13. Shadowgraph of projectile moving at $2.2 \mathrm{~km} / \mathrm{s}$ in propane, giving a normal shock density ratio of 17 . Again the features are like those in Fig. 12 and in stark contrast to the smooth shocks of Figs. 10 and 11. 


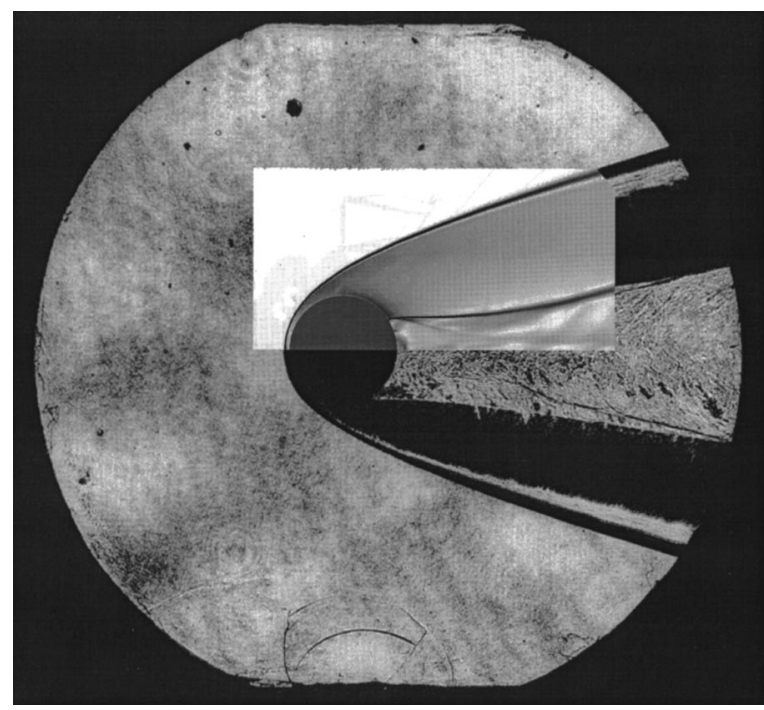

FIG. 14. Overlay of shadowgraph of Fig. 10 with pseudo-schlieren image of computation of flow over a sphere at $M_{\infty}=5.5$ and $\gamma=1.13$. The computational image, unlike the experimental one, is not line-of-sight integrating, so that it shows the details of the central wake much more pronouncedly. The disturbance at the inflow boundary is of the same form as that in the exploratory computations at an amplitude of $0.5 \%$.

pends on both the Mach number and the specific heat ratio, a given density ratio permits the choice of the right Mach number. Using the same computational setup as for the exploratory experiment, the flow over a sphere was computed for conditions as near as possible to those in Figs. 10 and 12. The results are shown in Figs. 14 and 15 as overlays over the experimental shadowgraphs. It is important to note that, in both cases, the disturbance introduced at the inflow boundary was the same. Clearly this disturbance is damped in the low density ratio case and amplified in the high density ratio flow.

\section{CONCLUSIONS}

A computational study of the stability of the shear layer formed by the curved bow shock in the shock layer of a hypervelocity flow over a blunt body revealed the following results:

(1) In plane flow over a cylinder-wedge, the shear layer is separated from the body by a roughly parallel region in which the flow is supersonic relative to the region of maximum shear.

(2) This layer is unstable to disturbances introduced in the free stream, when the disturbance wavelength is of the order of the nose radius.

(3) The structures formed by the instability grow by two nonlinear mechanisms. First, they push oblique shock waves into the flow beneath them that reflect from the body and reinforce them. Second, they perturb the bow shock, forming triple points from which shear layers issue that feed the shear layer.

(4) In axisymmetric flow over a sphere-cone, the shear layer is much closer to the body, so that the structures do not move supersonically with respect to the flow between

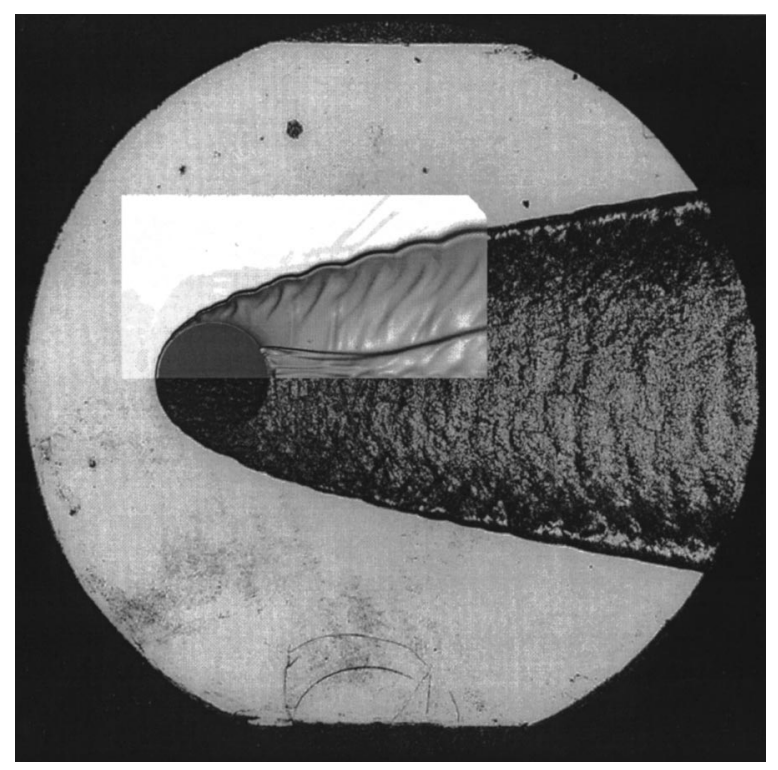

FIG. 15. Overlay of shadowgraph of Fig. 12 with pseudo-schlieren image of computation of flow over a sphere at $M_{\infty}=10$ and $\gamma=1.07$. The disturbance amplitude is again $0.5 \%$. Note the similarity between the experimental and computational shock perturbations.

them and the body, so that the dominant nonlinear mechanism of growth is through the perturbation of the bow shock by the structures.

(5) A physical experiment with propane and carbon dioxide flow over a sphere confirms the computational results, giving very good agreement, and confirming the existence of a critical normal shock density ratio of around 14.

The existence of a critical density ratio, beyond which the shock layer becomes unstable, shows that the simple Newtonian theory for hypersonic flow at high density ratio may be safely applied only when the normal shock density ratio is less than about 14 . This study is an example of how a relatively inexpensive exploratory computational study may be used to design an experiment, which, were it necessary to do the exploratory study experimentally, would be very expensive indeed. With this approach, the expense of the physical experiment can be kept to a minimum.

${ }^{1}$ M. J. Lighthill, "Dynamics of a dissociating gas," J. Fluid Mech. 2, 1 (1957).

${ }^{2}$ W. D. Hayes and R. F. Probstein, Hypersonic Flow Theory (Wiley, New York, 1959).

${ }^{3}$ P. G. Drazin and W. H. Reid, Hydrodynamic Stability (Cambridge University Press, Cambridge, 1981).

${ }^{4}$ M. Zhuang, P. E. Dimotakis, and T. Kubota, "The effect of walls on a spatially growing supersonic shear layer," Phys. Fluids 2, 599 (1990).

${ }^{5}$ K. F. Stetson, E. R. Thompson, J. C. Donaldson, and L. G. Siler, "Laminar boundary layer stability experiments on a cone at Mach 8, Part 2: Blunt cone," AIAA Paper No. 84-0006 (1984).

${ }^{6}$ A. Vaszonyi, "On rotational flows," Q. Appl. Math. 3, 29 (1945).

${ }^{7}$ J. J. Quirk, "Amrita-A computational facility (for CFD modeling)," VKI 29th CFD Lecture Series (von Kármán Institute for Fluid Dynamics, Brussels, 1998).

${ }^{8}$ P. Lemieux, "The instability of shear layers produced by curved shocks," Ph.D. thesis, Caltech (1999).

${ }^{9}$ J. Bélanger, M. Kaneshige, and J. E. Shepherd, "Detonation initiation by 
hypervelocity projectiles," in Shock Waves, Proceedings of the 20th International Symposium on Shock Waves, edited by B. Sturtevant, J. E. Shepherd, and H. G. Hornung (World Scientific, Singapore, 1995).

${ }^{10}$ W. C. Reynolds, "The element potential method of chemical equilibrium analysis: Implementation in the interactive program STANJAN," Techni- cal Report, Department of Mechanical Engineering, Stanford University (1986).

${ }^{11} \mathrm{G}$. K. Tumakaev, "Kinetic phase transition in molecular gases and its effect on flow formation behind a detached shock wave," J. Tech. Phys. 52, 690 (1982). 